\title{
NEW DIMENSIONLESS NUMBER TO PREDICT CAVITATION IN ACCELERATED FLUID
}

\author{
G. GARCIA-ATANCE FATJO \\ Jost Institute for Tribotechnology, University of Central Lancashire, UK.
}

\begin{abstract}
Cavitation is the formation of vapour cavities in a liquid due to a local low pressure. The traditional cavitation number is used to predict the occurrence of cavitation in liquid flows through devices such as pumps, propellers or dam spillways. However, this number can only be applied when cavitation is produced by changes in the dynamic and static pressure in a liquid flow. There are other means to produce cavitation where the traditional cavitation number cannot be applied. The purpose of this research is to formulate a new dimensionless number valid to predict cavitation in some scenarios where the traditional cavitation number fails. The 'tube-arrest' method produces cavitation by subjecting a column of liquid to a high acceleration without the need of any velocity between the liquid and the tube. In this scenario, the traditional number is not useful due to the low values of relative velocity between liquid and walls. However, the dimensionless number reported here predicts accurately the occurrence of cavitation in the 'tube-arrest' method, as it is shown by Finite Element Method analysis. There is another scenario where the dimensionless number is tested successfully; that is, in the bulk of a liquid downstream of a closing valve. A systematic comparison between the values of the dimensionless number and the occurrence of cavitation predicted by the FEM analysis is given. On the other hand, the values of the traditional cavitation number are calculated and it is shown that these values are meaningless in these scenarios. In contrast, the agreement between the prediction of the dimensionless number and the simulations is excellent. It is concluded that the new dimensionless number predicts cavitation in scenarios where the traditional number is meaningless.
\end{abstract}

Keywords: acceleration, cavitation, dimensionless, shock, tube arrest.

\section{INTRODUCTION}

There is a limited list of dimensionless numbers that are used to predict the behaviour of fluids. One of the most well known is the Reynolds number that was first used in 1883 [1] and is commonly used in many daily life applications to determine the turbulent, laminar or transient regime of the flow. Within these numbers, one that is of interest in this paper is the so-called cavitation number. It is the ratio of the drop of pressure needed for cavitation to the dynamic pressure. It was introduced by Thoma and Leraux around 1923-1925 [2]. It can be regarded as a special case of the pressure coefficient or a modified Eurler's number. It is used to measure the potential of a flowing system to cavitate. Its most common form is:

$$
N_{C A}=\frac{p-p_{v}}{1 / 2 \rho v^{2}}
$$

where $N_{C A}$ is the cavitation number, $p$ is the pressure of the undisturbed liquid, $p_{v}$ is the vapour pressure of the liquid, $\rho$ is the density of the liquid and $v$ is the velocity of the liquid. The liquid passing close to a propeller, an orifice or any disruption will be prone to cavitate if $N_{C A} \ll 1$.

Cavitation is the change from liquid to vapour due to a drop in pressure, as opposed to boiling that is due to a raise in temperature. Drastic local changes in pressure are common in flowing liquid systems where hydrodynamic effects can lead to pressure falls producing cavitation. Examples of this are propellers and pumps where cavitation may be present leading 
to loss of efficiency, noise and damage. In these cases, the traditional cavitation number can be used to predict cavitation. However, cavitation can also be produced by ultrasonic sound, vibration or acceleration. In these cases, the traditional cavitation number cannot be applied successfully because here a flowing system is not require to cavitate. The main reason for the drop in pressure in these cases is not a hydrodynamic effect of the flowing liquid around a corner or a curve but a change of pressure caused by a sonic wave, a vibrating movement, a shock or an acceleration.

In a liquid that is still, without any velocity, the value of cavitation number is infinite and suggests that there are no chances to develop cavitation. However, if it is subjected to an acceleration, a pressure wave is created and will develop cavitation in certain conditions. An example of this situation would be the 'tube-arrest' method where the inertial reference system is conveniently temporarily attached to the tube before it is arrested. Hence, either the tube or the liquid is still until an acceleration pulling downwards affects them.

Here, a new dimensionless number to predict cavitation, considering that the pressure drop is due to acceleration, is presented.

\section{FORMULATION}

The new dimensionless number is formulated as

$$
G_{A}=\frac{p-p_{v}}{\rho a L}
$$

where $G_{A}$ is the new dimensionless number, $p$ is the pressure of the liquid before or after being subjected to acceleration, $p_{v}$ is the vapour pressure of the liquid, $\rho$ is the density of the liquid, $L$ is the effective length of the liquid or column subjected to acceleration measured parallel to the acceleration vector and $a$ is the acceleration. The number is created in such a way that the liquid will cavitate if $G_{A} \ll 1$ for consistency with the cavitation number. The sense of the acceleration is important in order to produce a decrease of pressure rather than an increase of pressure.

A comparison with the traditional cavitation number of the new dimensionless number shows that the upper part of the ratio is the same, that is the pressure drop needed to cavitate, while the bottom part of the ratio differs. In the traditional cavitation number it is the dynamic pressure. In the new dimensionless number it is the change of pressure due to acceleration. It is derived from the 'rigid column theory', neglecting the viscosity, used in simple analysis of water hammers. According to this theory, the increment or decrement of pressure in the pipe is given by

$$
\Delta p=\rho \frac{d v}{d t} L
$$

The same relation can be obtained using the Euler's equation of motion for one-dimensional non-viscous incompressible fluid flow neglecting the action of the gravity and at constant cross-section.

Another difference between the traditional cavitation number and the new dimensionless number is that the former is applicable to steady flows while the latter is for unsteady flows.

An alternative way to write the new dimensionless number could be

$$
G_{A}=\frac{p-p_{v}}{\rho \frac{\Delta v}{\Delta t} L}
$$


where $\Delta v$ is the change of velocity in the time $\Delta t$. An approximation of the new dimensionless number when $p_{v} \ll p$ is:

$$
G_{A} \approx \frac{p}{\rho a L}
$$

The effective length $L$ of the column is normally the geometrical length of the water column. However, if the acceleration lasts a very short time, it should be considered the value of the length as $c \Delta t$ should this be smaller than the geometrical length, where $c$ is the speed of sound in the liquid and $\Delta t$ is the time the acceleration lasts. This consideration makes the new dimensionless number suitable for shocks of very short duration.

\section{SIMULATIONS AND RESULTS}

The author has looked for two practical cases where the new dimensionless number can be validated. They are shown in Fig. 1. The first one is the 'tube-arrest' method, introduced by Chesterman [3], that is a test to produce $a b$ initio cavitation in a controlled way [4-12]. The second one is the decrease of pressure in the bulk of the liquid in a horizontal pipe downstream of a closing valve. In the region upstream of the valve, there would be an increase of pressure when closing the valve and if the closure is abrupt, a water hammer is produced provided the pipe is long enough. In the region downstream of the valve, there would be a decrease of the pressure, and if the closure of the valve is fast enough, cavitation is produced provided the pipe is long enough. This second case should not be confused with the cavitation in the constriction formed by the valve while it is partially closed.

The simulation of pressure changes in the pipe and tube have been performed by the FEM. The equations solved are the 1D partial derivative equations for water hammer taking into account the change of speed of sound due to the elasticity of the pipe or tube. In the case of water flow in the pipe, the friction force is estimated by the use of a Darcy friction factor of 0.04 that is a typical value for a pipe with normal roughness, internal diameter $60 \mathrm{~mm}$ and 1

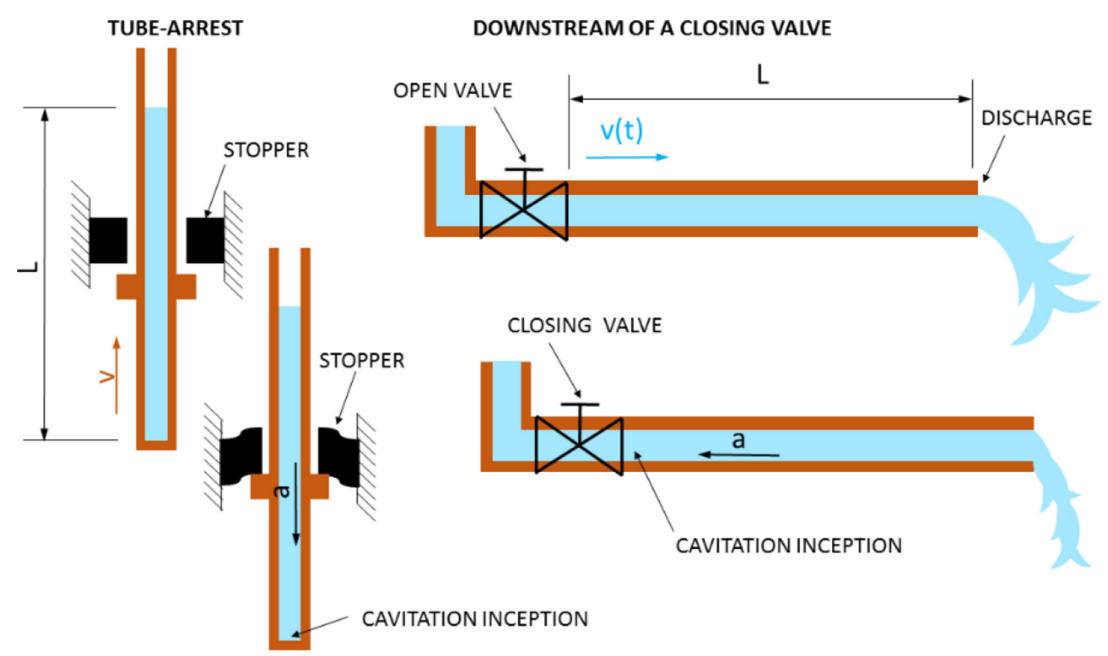

Figure 1: Diagram of scenarios. The 'tube-arrest' method is represented just before it is 'arrested' and while it is 'arrested'. The downstream of a valve system is represented before the valve is being closed and while it is being closed. Length, acceleration of the fluid and region of cavitation inception are indicated. 
$\mathrm{m} / \mathrm{s}$ average flow speed. In the case of the 'tube-arrest' method, a non-inertial reference frame is used attached to the tube. The criteria for the existence of cavitation during simulations is the reach of a local pressure smaller than the vapour pressure of the liquid. This criteria is commonly used in simulations to evaluate the presence of cavitation. In practice, the vapour pressure is very small and it is equivalent to reach a local negative pressure, that is the liquid would be in tension.

The 'tube-arrest' consists of a cylindrical vertical tube, partially filled with liquid, that is subjected to an upward movement and suddenly 'arrested'. Therefore, the liquid is subjected to a strong acceleration that produces a reduction of the pressure at the bottom of the column for a short time. The dimensionless number $G_{A}$ is calculated with the acceleration of the tube at the moment that is arrested, with the height of the liquid column as the length $L$ and with the sum of the ambient pressure and the hydrostatic pressure at the bottom of the column as the pressure $p$.

Figure 2 shows the contour lines of $G_{A}$ for the 'tube-arrest' method with water for a variety of accelerations and lengths. Lengths are from $3 \mathrm{~cm}$ to $100 \mathrm{~m}$. Accelerations are from 0.32 $\mathrm{ms}^{-2}$ to $10,000 \mathrm{~ms}^{-2}$. Further, Fig. 1 presents the combinations of acceleration and length where cavitation is obtained according to a simulation taking into account the compressibility of water and the elasticity of the tube. The hatched area are the conditions where water will

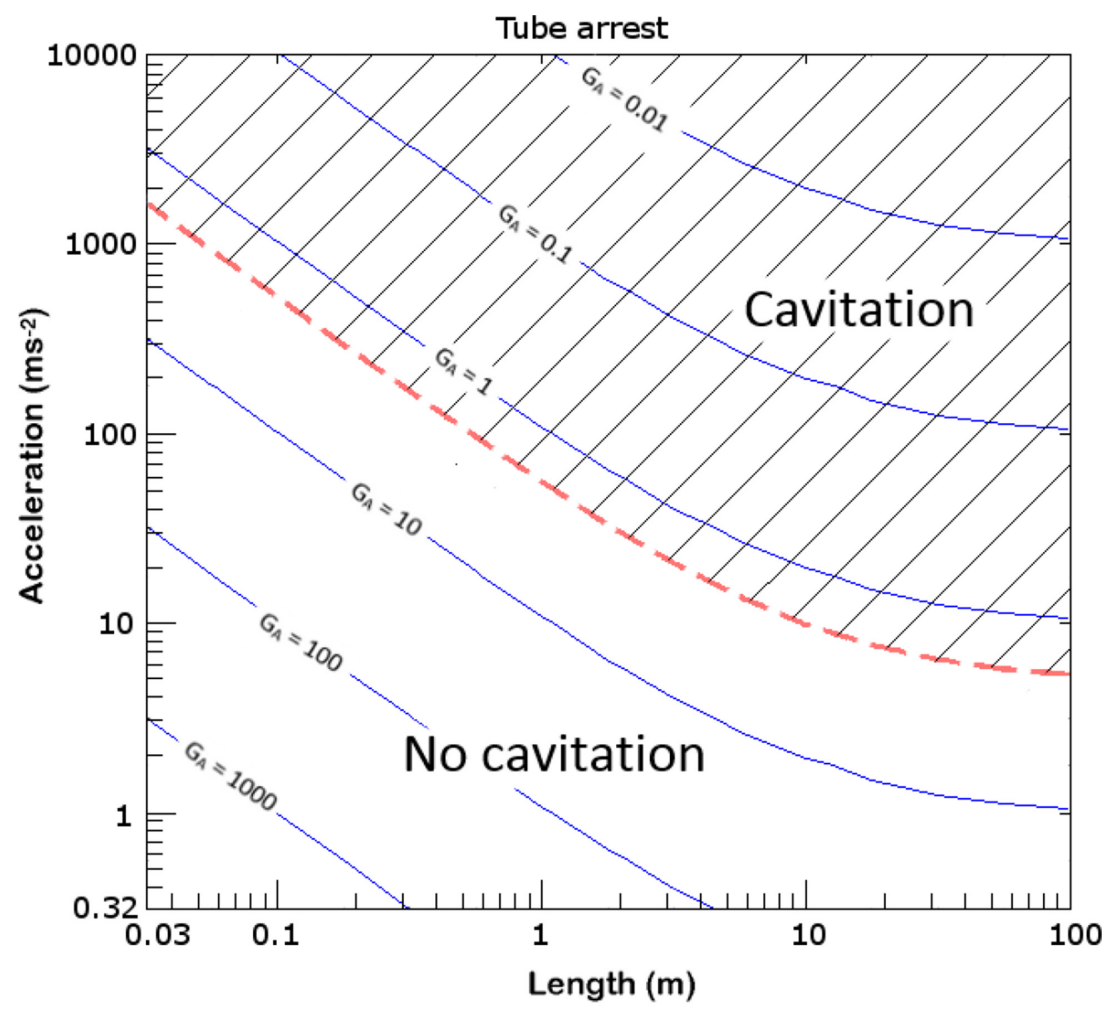

Figure 2: Contour lines and simulation for 'tube-arrest' method. Dimensionless number $G_{A}$ as a function of the acceleration and the length taking into account the hydrostatic pressure. Hatched area corresponds to the region where cavitation exists according to simulations. 


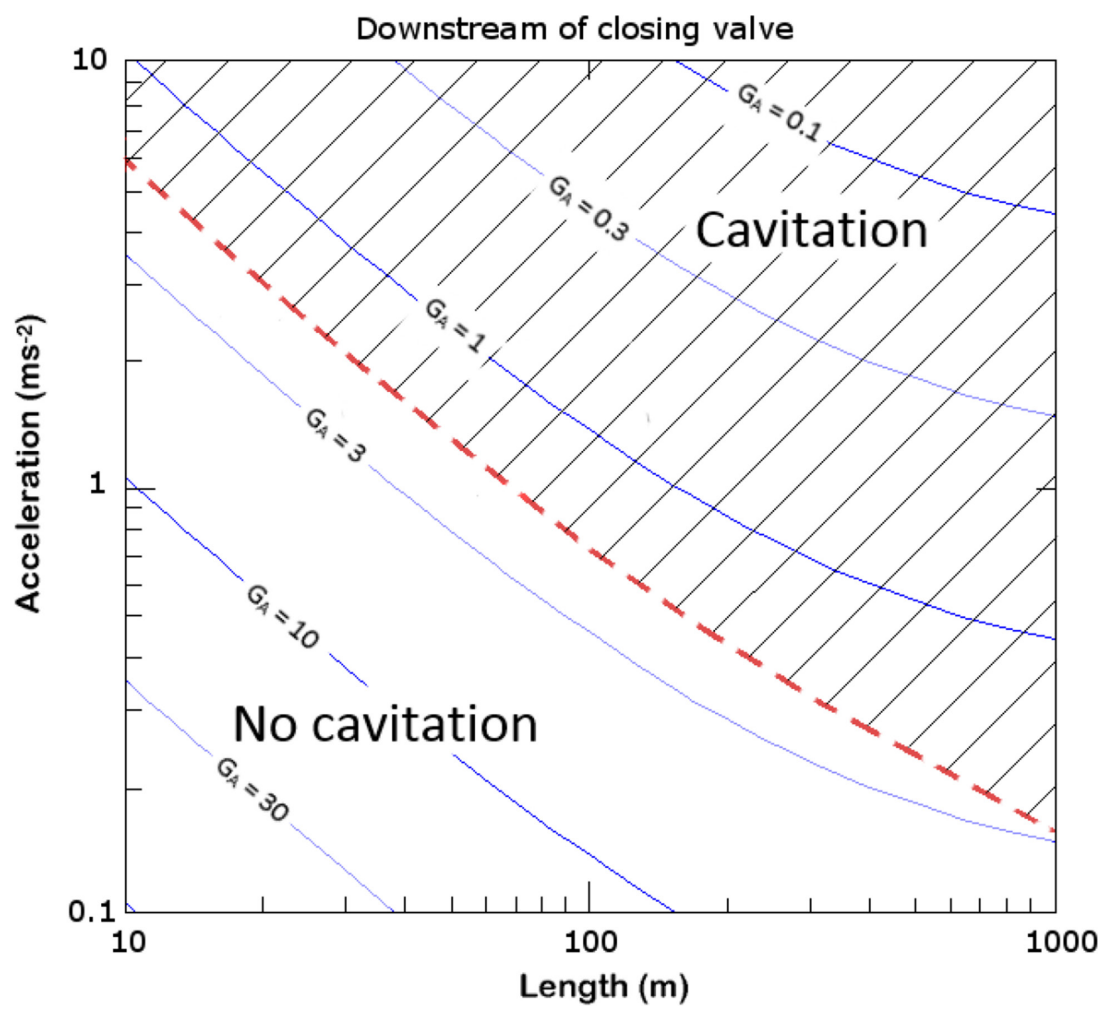

Figure 3: Contour lines and simulation for the bulk of the water downstream of a closing valve. Dimensionless number $G_{A}$ as a function of the acceleration and the length taking into account the pressure gradient along the pipe. Water at mean initial velocity of $1 \mathrm{~ms}^{-1}$. Internal pipe diameter is $60 \mathrm{~mm}$. Hatched area corresponds to the conditions where cavitation exists according to simulations.

cavitate. The red dashed line represents the limit of the existence of cavitation. The value of $G_{A}$ for the limit of existence of cavitation goes from 1.93 for length of $0.03 \mathrm{~m}$ to 1.99 for length of $100 \mathrm{~m}$.

Figure 3 shows the contour lines of $G_{A}$ for the bulk of the water downstream of a closing valve for a variety of accelerations and lengths. Lengths are from $10 \mathrm{~m}$ to $1,000 \mathrm{~m}$. Accelerations are from $0.1 \mathrm{~ms}^{-2}$ to $10 \mathrm{~ms}^{-2}$. Further, Fig. 3 presents the combinations of acceleration and length where cavitation is obtained according to a simulation taking into account the compressibility of water and the elasticity of the pipe. The hatched area shows the conditions where water will cavitate. The red dashed line represents the limit of the existence of cavitation. The value of $G_{A}$ for the limit of existence of cavitation goes from 1.80 for pipe length of $10 \mathrm{~m}$ to 2.81 for pipe length of $1,000 \mathrm{~m}$.

The limit of existence of cavitation in Fig. 3 is not parallel to the contour lines when the length of the pipe is long. This is explained with the fact that the values of $G_{A}$ have been calculated with the initial pressure of the liquid just downstream of the valve. This pressures includes the gradient pressure along the horizontal pipe due to the initial velocity and viscosity of the water. This increases the value of $p-p_{v}$ since $p$ is higher the longer the pipe 
is, and as a consequence, the contour lines are not straight lines. If the ambient pressure had been taken for the calculation of $G_{A}$, that is the final pressure when the water comes to rest, the contour lines would have been straight lines. It would have followed better the red dashed line. This happens because the simulations takes into account these two pressures: the initial pressure due to the pressure gradient as an initial condition and the ambient pressure at the end of the pipe where it discharges as a boundary condition. Consequently, cavitation is not hampered by the initial gradient pressure. Water cavitates at higher $G_{A}$ number in Fig. 3 at long lengths because this number is calculated with the built up pressure before closing the valve instead of the more favourable final ambient pressure. Had the new dimensionless number been calculated with the final ambient pressure the agreement would have been better in the bottom right corner of Fig. 3.

\section{DISCUSSION}

The comparison of the traditional cavitation number values with the values of the new dimensionless number follows. For the case of the downstream of a closing valve system, the traditional cavitation number value is 198 for all range of conditions represented in Fig. 3 at room pressure and temperature. The number does not change and is unable to predict cavitation. Indeed, the traditional number takes into account the initial velocity of the fluid and neglects the transient situation that causes cavitation. Taking an intermediate velocity during this transient makes the traditional cavitation number even bigger. For the existence of cavitation the traditional cavitation number should be smaller than 1 but this is only valid if the cavitation is produced as a consequence of certain value of dynamic pressure and existence of turbulence. As explained before, the cavitation is produced by a wave pressure due to the acceleration the fluid is subjected to, that is a transient.

For the 'tube-arrest' method, the situation is similar to the one just explained above. The actual velocity of the fluid with respect to the walls of the tube is 0 . Hence, the traditional cavitation number is infinite and is unable to predict cavitation. The discussion should be finished here. However, somebody might claim that the travelling speed of the tube should be taken into account for this calculation since when the tube is arrested, it stops and the liquid continues moving upwards at this speed. This is not correct as cavitation happens at the bottom of the tube rather than along it. However, in order to double check, this speed is taken and the values of the traditional cavitation number when cavitation starts are 188 when the length of the tube is $100 \mathrm{~m}$, and 1990 for length $0.03 \mathrm{~m}$. The values are much bigger than 1 and have no significance in the prediction of cavitation as explained before.

A further comparison of the values of the traditional cavitation number and the new dimensionless number is given in Table 1. The table presents the values for both dimensionless numbers for a hydrofoil at two speeds, for the 'tube-arrest' method arrested with two different accelerations and for a variation of the 'tube-arrest' method where the tube is initially stationary and then it is propelled downwards.

Further refinement looking at the simulations presented here show that in case they had been done assuming incompressibility of the water and perfectly rigid pipe or tube, the limit of cavitation according to those simulations would have been approximately coincidence with the contour line $G_{A} \approx 1$. However, the more realistic simulation used here makes the limit of cavitation approximately around $G_{A} \approx 2$. This is explained by looking at the pressure in the bottom of the tube and/or downstream the valve along the time. At the beginning, the pressure in this point is the initial pressure and when acceleration starts, this pressure decreases progressively. In the simplistic model assuming incompressibility the reduction of pressure is 
Table 1: Comparison of the traditional cavitation number with the new dimensionless number for different scenarios. Cavitation would exist when the applicable number (underlined) is smaller than 1

\begin{tabular}{|c|c|c|c|}
\hline Case & $\mathbf{N}_{\mathbf{C A}}$ & $\mathbf{G}_{\mathbf{A}}$ & Comment \\
\hline $\begin{array}{l}\text { Hydrofoil travelling } \\
\text { at } 2 \mathrm{~m} / \mathrm{s} \text { at a depth } \\
\text { of } 1 \mathrm{~m} .\end{array}$ & 54 & $\infty$ & $\begin{array}{l}\text { NO CAVITATION } \\
\text { According to } \underline{\mathbf{N}}_{\mathbf{C A}} \text { it does not cavitate. } \mathrm{G}_{\mathrm{A}} \text { does } \\
\text { not work as it is a steady situation and there is } \\
\text { no change of velocity. }\end{array}$ \\
\hline $\begin{array}{l}\text { Hydrofoil travelling } \\
\text { at } 30 \mathrm{~m} / \mathrm{s} \text { at a depth } \\
\text { of } 1 \mathrm{~m} .\end{array}$ & 0.24 & $\infty$ & $\begin{array}{l}\text { CAVITATION } \\
\text { According to } \underline{\underline{\mathbf{N}}}_{\mathbf{C A}} \text { it cavitates. Again, } \mathrm{G}_{\mathrm{A}} \text { gives an } \\
\text { infinite number that is meaningless for this case. }\end{array}$ \\
\hline $\begin{array}{l}\text { 'Tube-arrest' method } \\
\text { with water column } \\
\text { of } 1 \mathrm{~m} \text { length, ar- } \\
\text { rested with } 3 \mathrm{~m} / \mathrm{s}^{2} \\
\text { acceleration. }\end{array}$ & $\begin{array}{l}870 \\
\left(\mathrm{v}_{0}=0.5\right. \\
\mathrm{m} / \mathrm{s}) \\
21,767 \\
\left(\mathrm{v}_{0}=0.1\right. \\
\mathrm{m} / \mathrm{s})\end{array}$ & 0.1 & $\begin{array}{l}\text { CAVITATION (checked in simulation) } \\
\text { According to } \underline{\mathbf{G}}_{\mathbf{A}} \text { it cavitates. } \mathrm{N}_{\mathrm{CA}} \text { is infinite } \\
\text { if the right velocity }(\mathrm{v}=0) \text { is computed (the } \\
\text { velocity of the fluid respect the tube wall); } \\
\text { however, there could be some arguement that } \\
\text { the traveling velocity of the liquid and the wall } \\
\text { before arresting should be taken. Then for the } \\
\text { cases with the travelling velocity indicated on } \\
\text { the left the numbers of } \mathrm{N}_{\mathrm{CA}} \text { are meaningless } \\
\text { since they indicate no cavitation although the } \\
\text { simulations show cavitation clearly. }\end{array}$ \\
\hline $\begin{array}{l}\text { 'Tube-arrest' method } \\
\text { with water column } \\
\text { of } 1 \mathrm{~m} \text { length, ar- } \\
\text { rested with } 0.5 \mathrm{~m} / \mathrm{s}^{2} \\
\text { acceleration. }\end{array}$ & $\begin{array}{l}870 \\
\left(\mathrm{v}_{0}=0.5\right. \\
\mathrm{m} / \mathrm{s}) \\
21,767 \\
\left(\mathrm{v}_{0}=0.1\right. \\
\mathrm{m} / \mathrm{s})\end{array}$ & 35 & $\begin{array}{l}\text { NO CAVITATION (checked in simulation) } \\
\text { According to } \mathbf{G}_{\mathbf{A}} \text { it does not cavitate. } \mathrm{N}_{\mathrm{CA}} \text { is } \\
\text { infinite if the right velocity }(\mathrm{v}=0) \text { is computed } \\
\text { (the velocity of the fluid respect the tube wall) } \\
\text { as in the previous case is also checked with } \\
\text { the travelling velocities before being arrested } \\
\text { indicated in parenthesis. } \mathrm{N}_{\mathrm{CA}} \text { are meaningless } \\
\text { since the value is random, it depends on the } \\
\text { random initial velocity that actually depends } \\
\text { on the inertial reference system used as shown } \\
\text { with the next example. }\end{array}$ \\
\hline $\begin{array}{l}\text { Stationary water } \\
\text { column }(0.1 \mathrm{~m} \\
\text { length) in a tube that } \\
\text { suffers a shock } \\
\text { downwards of } 3.5 \mathrm{~m} / \\
\mathrm{s}^{2} \text {. Variation of } \\
\text { 'tube-arrest' method. }\end{array}$ & $\infty$ & 0.32 & $\begin{array}{l}\text { CAVITATION (checked in simulation) } \\
\text { According to } \underline{\mathbf{G}}_{\mathbf{A}} \text { it cavitates. } \mathrm{N}_{\mathrm{CA}} \text { is infinite } \\
\text { and is meaningless since it indicates no } \\
\text { cavitation although the simulations show } \\
\text { clearly cavitation. }\end{array}$ \\
\hline
\end{tabular}

instantaneous rather than progressive. As a consequence, the realistic model including compressibility, gives a much smaller reduction of the pressure initially. This smaller reduction is 'recovered' later by achieving temporally an even bigger reduction, lower minimum pressure, 
in order to stop the liquid travelling upwards or forwards that is the final result in both simulations. This explains why the limit of existence of cavitation is for values $G_{A} \approx 2$ instead of 1 .

Now that it is demonstrated that the new dimensionless number works, it is needed to define its limits. The simulations suggest that for $G_{A}<2$ cavitation exists. However, in practice, it would be difficult to measure or estimate the acceleration accurately. Furthermore, the acceleration is unlikely to be constant. The simulations have taken constant acceleration for simplicity and for consistency between the two cases. Besides, practical cases may have a more complex geometry than a pipe or a tube and the dimensionless number should still be valid for these containers. Nevertheless, these types of limitations are very common in most dimensionless numbers and they are used successfully. A conservative approach would be:

- When $G_{A} \ll 1$, cavitation is expected.

- When $G_{A} \gg 3$, cavitation due to acceleration is not expected.

\section{CONCLUSION}

A new dimensionless number different from the traditional cavitation number is presented here. Comparisons between the two numbers are done and explanations of the different results are given. The new dimensionless number accurately predicts cavitation in transient conditions due to acceleration. This cannot be done by the traditional number.

\section{REFERENCES}

[1] Reynolds, O., An experimental investigation of the circumstances which determine whether the motion of water shall be direct or sinuous, and of the law of resistance in parallel channels. Philosophical Transactions of the Royal Society of London, 174, pp. 935-982, 1883.

[2] Franc, J.-P. \& Michel, J.-M., Fundamentals of cavitation. Fluid mechanics and its applications. Volume 76. ISBN: 978-1-4020-2232-6 (Print) 978-1-4020-2233-3 (Online) p13, 2005.

[3] Chesterman, W.D., The dynamics of small transient cavities. Proceeding of the Physical Society. Section B, 65, pp. 846-858, 1952.

http://dx.doi.org/10.1088/0370-1301/65/11/302

[4] Qi-Dai, C. \& Long, W., Production of large size single transient cavitation bubbles with tube arrest method. Chinese Physics, 13(4), pp. 564-570, 2004.

http://dx.doi.org/10.1088/1009-1963/13/4/028

[5] Overton, G.D.N. \& Trevana, D.H., Cavitation phenomena and the occurrence of pressure-tension cycles under dynamic stressing. Journal of Physics D: Applied Physics, 14, pp. 241-250, 1981. http://dx.doi.org/10.1088/0022-3727/14/2/016

[6] Williams, P.R., Williams, P.M. \& Brown, S.W.J., Pressure waves arising from the oscillation of cavitation bubbles under dynamic stressing. Journal of Physics D: Applied Physics, 30, pp. 1197-1206, 1997. http://dx.doi.org/10.1088/0022-3727/30/8/007

[7] Williams,P.R.,Williams, P.M.\&Brown, S.W.J.,Aninstrument for studying cavitation phenomena in liquids subjected to tension generated ab ignition and by free-surface refection of compressional waves. Measurement Science and Technology, 9, pp. 976-982, 1998a. http://dx.doi.org/10.1088/0957-0233/9/6/015 
[8] Williams, P.R., Williams, P.M., Brown, S.W.J., Tensile properties of liquid mercury under pulsed dynamic stressing. Journal of Physics D: Applied Physics, 31, pp. 1923-1926, 1998. http://dx.doi.org/10.1088/0022-3727/31/15/023.

[9] Williams, P.R., Williams, P.M., Brown, S.W.J., A study of liquid jets formed by bubble collapse under shock waves in elastic and Newtonian liquids, Journal of Non-Newtonian Fluid Mechanics, 76(1-3), 1998, pp. 307-325, 1998. http://dx.doi.org/10.1016/S0377-0257(97)00124-9

[10] Williams, P.R., Williams, P.M., Brown, S.W.J. \& Papadopoulou, K., Dynamic stressing of a liquid-liquid interface by tension. Journal of Physics D: Applied Physics, 33, pp. 1-7, 2000.

http://dx.doi.org/10.1088/0022-3727/33/1/301

[11] Chong-Fu, Y., Chao, Li., De-Long, X. \& Jing-Jun, D., The pressure field in the liquid column in the tube-arrest method. Chinese Physics B, 17(7), pp. 2580-2589, 2008. http://dx.doi.org/10.1088/1674-1056/17/7/040

[12] Chongfu, Y. \& Chao, L., Onset of cavitation by the strong tension spike from a tubearrest apparatus. Science China Physics, Mechanics and Astronomy, 2010, 53(2), pp 301-305, 2010.

http://dx.doi.org/10.1007/s11433-010-0130-1 